Proc. of 12th International Workshop on Positron and Positronium Chemistry, August 28-September 1, 2017, Lublin, Poland

\title{
Ortho-Positronium Annihilation in Room Temperature Ionic Liquids
}

\begin{abstract}
T. HIRADE*
${ }^{a}$ Nuclear Science and Engineering Center, Japan Atomic Energy Agency, Tokai, 319-1195 Japan

${ }^{b}$ Institute of Quantum Beam Science, Ibaraki University, 4-12-1 Narusawa, Hitachi, Ibaraki, 316-8511 Japan

Very new phenomena have been found in room temperature ionic liquids, e.g. oscillation of positronium (Ps) bubble. It is almost impossible to see this kind of phenomenon in usual molecular liquids. The Ps bubble oscillations in $N, N, N$-trimethyl- $N$-propylammonium bis(trifluoro-methanesulfonyl)imide (TMPA-TFSI), $N$ methyl- $N$-propylpiperidinium bis(trifluoromethane-sulfonyl) imide (PP13-TFSI) and 1-ethyl-3-methylimidazolium thiocyanate (EMIM-SCN) were successfully observed. Even though the Ps bubble size is smaller for EMIM-SCN, the oscillation frequency of the Ps bubble at $10^{\circ} \mathrm{C}$ above the melting temperatures was quite similar for the other room temperature ionic liquids. Hence, the Ps bubble oscillation is, probably, caused by the repulsive force initiated by the ionic structures in room temperature ionic liquids that can remain at $10-15^{\circ} \mathrm{C}$ above the melting temperatures.
\end{abstract}

DOI: 10.12693/APhysPolA.132.1470

PACS/topics: $78.70 . \mathrm{Bj}$

\section{Introduction}

Positronium (Ps) is a bound state of an electron and a positron. They have their own spin states therefore Ps has two states, singlet Ps (para-Ps; $p$-Ps) and triplet Ps (ortho-Ps; $o$-Ps). $p$-Ps and $o$-Ps have 125 ps and $142 \mathrm{~ns}$ intrinsic annihilation lifetime in vacuum, respectively. $o$ Ps lifetime, $142 \mathrm{~ns}$, is much longer and the positron in $o$-Ps has larger possibility to annihilate with electrons in surrounding molecules. This annihilation process is called "pick-off annihilation" and the lifetime is shortened to $1-5 \mathrm{~ns}$ in condensed materials. Some of the positrons do not form Ps and annihilate with one of the electrons in surrounding molecules with the lifetime of about 400 ps. It is free from Ps formation so-called "free positron" that does not mean a freely moving positron. Injected positrons in insulating materials will have possibility of Ps formation. The Ps formation in insulating materials is caused by the spur reaction [1] and the reaction between pre-existing trapped electrons and positron after its diffusion [2]. Ps has a negative work function in insulating materials and then Ps prefers to go out from the surface. If there are enough vacant space for Ps in the materials, Ps localizes in it. In the case of liquids, Ps pushes surrounding molecules away to create vacant space that we call "Ps bubble" and localize in it. Ps bubble is usually smoothly formed within very short time as $10 \mathrm{ps}[3]$.

Some researches indicated that the excess electrons in room temperature ionic liquids (RTILs) move freely for relatively long time [4]. Positron annihilation lifetime measurements of RTILs indicated that the first component lifetime was anomalously longer, i.e. 291 ps and 257 ps for TMPA-TFSI and 1-butyl-1-

\footnotetext{
*e-mail: hirade.tetsuya@jaea.go.jp
}

methylpyrrolidinium bis(trifluoromethanesulfonyl)imide (P14-TFSI), respectively, than the values in usual insulating materials [5]. Later, it was confirmed for some other RTILs [6]. Positron annihilation age-momentum correlation (AMOC) measurement is suitable way to know that the obtained anomalous long lifetimes for the first component was really the lifetime component from $p$-Ps intrinsic annihilation. The time resolved $S$ parameters could not be fitted with the anomalous long lifetimes for the $p$-Ps intrinsic annihilation but with the usual intrinsic $p$-Ps annihilation lifetime except for the smaller $S$ values at very young positron ages [5]. Therefore, it should be concluded that the anomalous long lifetimes obtained for RTILs must not be the $p$-Ps intrinsic annihilation lifetimes. They were just apparently obtained because of the non-exponentially decay curve at very young positron ages.

At first, the smaller $S$ values at very young positron ages were explained by the slower Ps formation [7] by referring the report indicating the excess electrons move freely for longer time [4]. However, detailed analysis of AMOC results, i.e. time resolved $W$-parameters indicated that Ps is squeezed at young positron ages in RTILs [7]. The reasonable explanation of the anomalously larger lifetimes of the first component was the slow Ps bubble formation, because of the smaller contact density of the positron and the electron of $p$-Ps in the squeezed Ps bubble.

The $o$-Ps pick off annihilation rates give the information of the size of the vacant space for Ps, i.e. Ps bubble size for liquids $[8,9]$. Hence, it was expected that the slow Ps bubble formation in RTILs could be detected by change of the $o$-Ps pick off annihilation rates. Unfortunately, there is the other annihilation from $P_{s}$ that is $p$-Ps intrinsic annihilation. This annihilation probability is larger than $o$-Ps pick off annihilation probability until about 400-600 ps. Possibility of the observation of the slow Ps bubble formation by the $o$-Ps pick off annihilation 
rates is just later than $400-600$ ps. Annihilation rate of free positron is constant and it would not be obstructive.

The method applied to extract the change of the $o$-Ps pick off annihilation rates was subtracting a simulated lifetime spectrum from measured spectrum. The simulated spectrum contains just several exponentially decaying curves, therefore non-exponential change of the annihilation rates can be separated. Then the oscillation of Ps bubble in RTILs was successfully observed [10]. The detail of the method was explained in Ref. [10].

Here, I will introduce the oscillation of $o$-Ps pickoff annihilation rates i.e. Ps bubble oscillation obtained for three RTILs and discuss what we can learn from the oscillation frequency.

\section{Experimental}

Positron annihilation lifetime measurements were performed by use of a set of two detectors consist of circular truncated cone shape (20 mm diameter at the top, $30 \mathrm{~mm}$ diameter at the bottom, and $20 \mathrm{~mm}$ height) $\mathrm{BaF}_{2}$ scintillators and H3378 (Hamamatsu) photomultiplier tubes. One detector was used for the start signal by detecting the $1.275 \mathrm{MeV}$ gamma-ray from ${ }^{22} \mathrm{Na}$ almost simultaneously with the time of a positron emission. The other detector was used for the stop signal by detecting one of the positron annihilation gamma-rays, $511 \mathrm{keV}$. The wave forms from the detectors were saved on the hard disk in a digital storage oscilloscope (DSO), Wavepro7100A (LeCroy) and the time interval between the start and the stop signals were analyzed afterward. The detail of the analysis can be found in Ref. [10]. It is needed to have good time resolution to detect high frequency oscillation. The time resolution was $150-160$ ps. Total count of every spectrum was about 3 million. PALSfit programme was used to analyze the positron annihilation lifetime spectra [11].

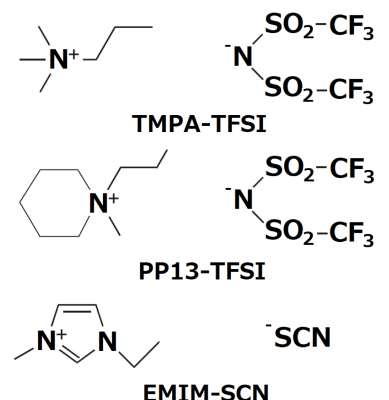

Fig. 1. Chemical structures of cations and anions of RTILs.

TMPA-TFSI (Kanto Chemical Co.), PP13-TFSI (Kanto Chemical Co.) and EMIM-SCN (Aldrich) were used without any purification in this study. The chemical structures are indicated in Fig. 1. Melting temperatures for TMPA-TFSI, PP13-TFSI and EMIM-SCN are $19^{\circ} \mathrm{C}$, $12^{\circ} \mathrm{C}$, and $-6{ }^{\circ} \mathrm{C}$, respectively.

\section{Results and discussion}

The oscillation frequencies of Ps bubble for TMPATFSI, PP13-TFSI and EMIM-SCN were obtained by the method explained above [10]. The oscillation component obtained for PP13-TFSI measured at $15.0^{\circ} \mathrm{C}$ is indicated in Fig. 2. The oscillation frequency was $7.35 \mathrm{GHz}$. Although it is already known that there exist high harmonics for Ps bubble oscillation [12], the line in Fig. 2 was calculated with just the fundamental frequency of $7.35 \mathrm{GHz}$.

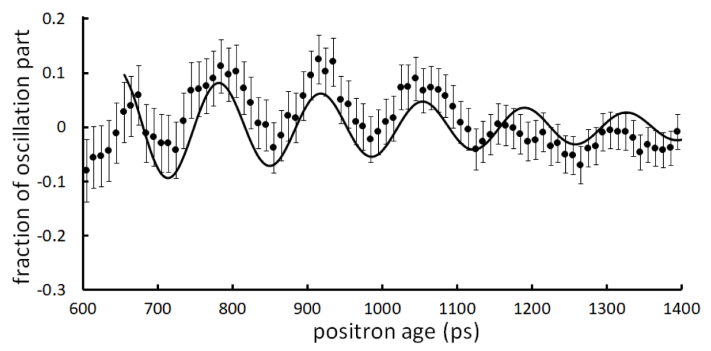

Fig. 2. Oscillation part obtained from positron annihilation lifetime spectrum measured at $15^{\circ} \mathrm{C}$ in $\mathrm{PP} 13-$ TFSI. The detail of the methods to obtain this result is indicated in [10].

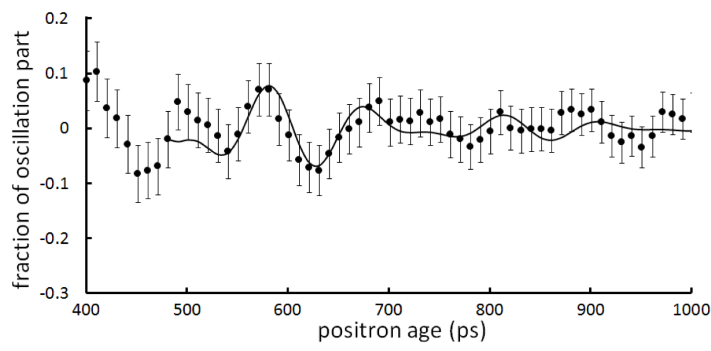

Fig. 3. Oscillation part obtained from positron annihilation lifetime spectrum measured at $3.4^{\circ} \mathrm{C}$ in EMIM$\mathrm{SCN}$. The detail of the methods to obtain this result is indicated in [10].

The oscillation component obtained for EMIM-SCN measured at $3.4{ }^{\circ} \mathrm{C}$ is indicated in Fig. 3. This temperature is about $10^{\circ} \mathrm{C}$ above the melting temperature of EMIM-SCN. It was reported that the oscillation of the $o$-Ps pick-off annihilation rate was observed for TMPATFSI at $30^{\circ} \mathrm{C}$ that is about $10^{\circ} \mathrm{C}$ above the melting temperature and could be reproduced with sum of the second and third higher harmonics [12]. The line in Fig. 3 was also obtained by the second and third higher harmonics of the fundamental frequency of $4.3 \mathrm{GHz}$. Although, the chemical structure of EMIM-SCN is very different from those of TMPA-TFSI and PP13-TFSI as indicated in Fig. 1, the oscillation of the $o$-Ps pick-off annihilation rate was successfully obtained.

The longest lifetimes, i.e. $o$-Ps pick-off annihilation lifetimes for PP13-TFSI at $20^{\circ} \mathrm{C}$, TMPA-TFSI at $30^{\circ} \mathrm{C}$, and EMIM-SCN at $3.4^{\circ} \mathrm{C}$ were $3.47,3.76$, and $1.98 \mathrm{~ns}$, respectively. According to the Tao-Eldrup formula [8, 9], that can be used to obtain the size of the Ps bubble, radii (volumes) of the Ps bubble in these RTILs are $0.39 \mathrm{~nm}$ $\left(0.26 \mathrm{~nm}^{3}\right), 0.41 \mathrm{~nm}\left(0.29 \mathrm{~nm}^{3}\right)$ and $0.28 \mathrm{~nm}\left(0.096 \mathrm{~nm}^{3}\right)$, respectively. These are the sizes of stable Ps bubbles in these RTILs observed at the tail part of the positron 
annihilation lifetime spectra. Although the volume of Ps bubble in EMIM-SCN is about $35 \%$ of the volumes in TMPA-TFSI and PP13-TFSI, Fig. 4 indicated that the oscillation frequency in EMIM-SCN at $3.4^{\circ} \mathrm{C}$ was almost on the same curve of the temperature dependence of the oscillation frequencies in PP13-TFSI and TMPA-TFSI. Here, the temperature means the difference between the measured temperatures and the melting temperatures.

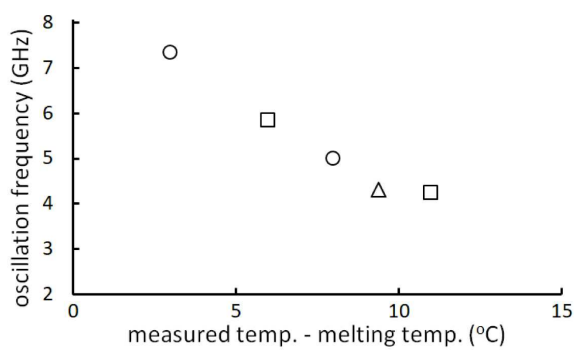

Fig. 4. Temperature dependence of the Ps bubble oscillation frequency. Horizontal axis indicates the temperature above the melting temperatures. $\bigcirc$ PP13TFSI, $\square$ TMPA-TFSI, $\Delta$ EMIM-SCN.

According to the study of bubble dynamics in liquids, bubble oscillation frequency should depend on the bubble radius [13]. The trigger of the oscillation is the Ps formation [10]. Ps has a negative work functions and then the Ps starts to push surrounding molecules and then the oscillation starts. This is quite like dynamic viscoelastic property measurements. As indicated above, (1) the bubble size does not affect the oscillation frequency, (2) the oscillation frequency just depends on the temperatures from melting temperatures. (1) can indicate that surface tension is not the source of the repulsive force for the Ps bubble oscillation in RTILs, (2) can indicate that the Ps bubble oscillation is caused by the structures remaining even above melting temperatures.

Synchrotron X-ray diffraction studies indicated that surface crystallization at the vapor-liquid interface of the ionic liquid (1-butyl-3-methylimidazolium hexafluorophosphate) is observed as sharp Bragg reflections even at $37^{\circ} \mathrm{C}$ above the melting temperature. It indicated the presence of a surface nanocrystallization of a RTIL even above the melting temperature [14]. It is indicating that the structure caused by the ionic interaction can remain even above the melting temperatures.

The structure by ionic interaction probably can remain even above melting temperatures and hence the repulsive force causing the Ps bubble oscillation might be the structure caused by the ionic interaction. The oscillation of $o$-Ps pick-off annihilation rate could be reproduced by the cosine curve initiated at time zero, i.e. almost same as the Ps formation time [10]. It means that the oscillation frequencies are quite stable even during the bubble expansion. The Ps bubble should be formed at energetically easier parts, i.e. probably non-ionic parts [15]. The nonionic parts are of course covered by ionic parts. When Ps pushes surrounding molecules, non-ionic parts can move easily and there will be no repulsive force. However, at the next of the non-ionic parts, there must be ionic parts and then the repulsive force that is proportional to the displacement of the ionic parts should appear. The repulsive force divided by the displacement is the modulus. The surface tension is not the cause of the repulsive force as mentioned above and the modulus caused by the ionic structure is, probably, the cause of the repulsive force to initiate the Ps bubble oscillation. The oscillation frequency depends on the modulus of the ionic parts therefore it is quite stable during the Ps bubble expansion and does not depends on the size of the Ps bubble.

\section{Conclusion}

The oscillations of $o$-Ps pick-off annihilation caused by the Ps bubble oscillations in TMPA-TFSI, PP13TFSI and EMIM-SCN were successfully observed. Even though the Ps bubble size is much smaller for EMIM$\mathrm{SCN}$, the fundamental frequencies of the Ps bubble oscillation at $10^{\circ} \mathrm{C}$ above the melting temperatures were quite similar for these three RTILs. Hence, I can conclude following things. (1) The repulsive force caused by the ionic structures is, probably, the reason of the Ps bubble oscillation. (2) The structure caused by the ionic interactions in bulk RTILs can remain even above the melting temperatures.

\section{Acknowledgments}

This research was supported by a Ministry of Education, Culture, Sports, Science and Technology Grant-inAid for Scientific Research (C), 16K05026, 2016-2018.

\section{References}

[1] O.E. Mogensen, J. Chem. Phys. 60, 998 (1974).

[2] T. Hirade, F.H.J. Maurer, M. Eldrup, Radiat. Phys. Chem. 58, 465 (2000).

[3] S.V. Stepanov, M.K. Mikhin, D.S. Zvezhinskii, V.M. Byakov, Radiat. Phys. Chem. 76, 275 (2007).

[4] R. Katoh, Y. Yoshida, Y. Katsumura, K. Takahashi, J. Phys. Chem. B 111, 4770 (2007).

[5] T. Hirade, Mater. Sci. Forum 607, 232 (2009).

[6] G. Dlubek, Y. Yu, R. Krause-Rehberg, W. Beichel, S. Bulut, N. Pogodina, I. Krossing, Ch. Friedrich, J. Chem. Phys. 133, 124502 (2010).

[7] T. Hirade, T. Oka, J. Phys. Conf. Series 443, 012060 (2013).

[8] S.J. Tao, J. Chem. Phys. 56, 5499 (1972).

[9] M. Eldrup, D. Lightbody, J.N. Sherwood, Chem. Phys. 63, 51 (1981).

[10] T. Hirade, JJAP Conf. Proc. 2, 011003 (2014).

[11] P. Kirkegaard, J.V. Olsen, M. Eldrup, N.J. Pedersen, PALSfit: A computer program for analysing positron lifetime spectra, Ris $\varnothing-\mathrm{R}-1652(\mathrm{EN}), 2009$.

[12] T. Hirade, J. Phys. Conf. Series 618, 012004 (2015).

[13] C.E. Brennen, Cavitation and Bubble Dynamics, Oxford University Press, 1995.

[14] Y. Jeon, D. Vaknin, W. Bu, J. Sung, Y. Ouchi, W. Sung, D. Kim, Phys. Rev. Lett. 108, 055502 (2012).

[15] J.N.C. Lopes, M.F.C. Gomes, A.A.H. Pádua, J. Phys. Chem. B Lett. 110, 16816 (2006). 